Volume 8, No.1.6, 2019

International Journal of Advanced Trends in Computer Science and Engineering

Available Online at http://www.warse.org/IJATCSE/static/pdf/file/ijatcse3581.62019.pdf

https://doi.org/10.30534/ijatcse/2019/3581.62019

\title{
Resolving the Effect of Phase Errors on Ultrafast Ultrasound Plane Wave Imaging
}

\author{
Asraf Mohamed Moubark ${ }^{1}$, Luzhen $\mathrm{Nie}^{2}$, Sawal Md Ali ${ }^{1}$, Mohd Hairi Mohd Zaman ${ }^{1}$, Steven Freear ${ }^{2}$ \\ ${ }^{1}$ Center for Integrated Systems Engineering and Advanced Technologies, Faculty of Engineering and Built \\ Environment, Universiti Kebangsaan Malaysia, Malaysia, \\ asrafmohamed@ukm.edu.my \\ ${ }^{2}$ Ultrasound Group, School of Electronic and Electrical Engineering, University of Leeds, United Kingdom,
} S.Freear@leeds.ac.uk

\begin{abstract}
The phase error caused by non-ideal physical transducer characteristics can affect the ultrasonic wave front during beamforming. If not compensated, the phase error can degrade the focusing capability and reduce the B-mode image spatial resolution. In this paper, the effect of the phase error on ultrafast coherent plane wave imaging (CPWI) was investigated using a bespoke ultrasound imaging system, ultrasound array research platform II (UARP II). The phase deviations between the aligned radio frequency (RF) signals from all active elements were calculated by applying crosscorrelation techniques with the RF signal from the first active element as the registration reference. A sign-reversed lag technique has been applied to the RF signals to encounter the phase error. When the phase error was corrected, the lateral resolution at $-6 \mathrm{~dB}$ and $-20 \mathrm{~dB}$ showed improvements from $0.42 \mathrm{~mm}$ to $0.34 \mathrm{~mm}$ and from $1.18 \mathrm{~mm}$ to $0.56 \mathrm{~mm}$, respectively. No significant changes occurred for the axial main lobe.
\end{abstract}

\section{INTRODUCTION}

The phase error is a terminology used to describe the deviation between the aligned radio frequency (RF) echo from each element during ultrasound beamforming. This happens mainly due to two reasons. Firstly, it is because of the non-ideal physical transducer characteristics and the second reason is the non-uniform speed of sound in the imaging medium [1-2]. The characteristics of each transducer are defined by a fixed central frequency, limited bandwidth, damping and sensitivity. Each transducer element is unique and none of them are same [3-4]. At the same time, not all elements in a transducer respond or vibrate equally and there is a deviation in between of them [1].

In an ideal case, it is expected the calculated time delays added to the received RF signals will align those signals before coherent summation [5-10]. However, in a real scenario the aligned RF signals are not always in phase due to the above reasons [11-13]. Many techniques have been proposed to estimate the phase error in ultrasound B-mode imaging. Some of the estimation techniques are the speckle brightness method [1] and the autocorrelation technique [14].

In this paper, only the phase error caused by the non-ideal physical transducer characteristics will be considered and compensated. But the proposed solutions, adding a signreversed lag to the RF signals can be applied to the correction of phase errors caused by the varied speed of sound as well.

\section{MATERIAL AND METHOD}

\subsection{Experimental Setup}

In order to analyse the effect of the phase error on plane wave imaging (PWI), a study was conducted on the wire targets located inside the degassed and deionized water as shown in Figure 1.

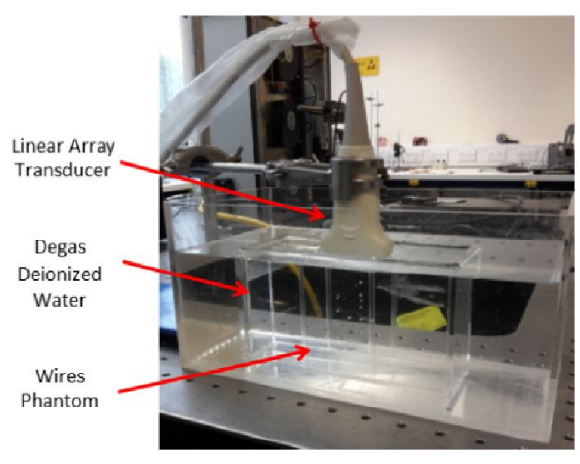

Figure 1: Experimental setup to scan wires phantom inside degassed and deionized water.

Performing ultrasound imaging in water is an advantage since the medium attenuation is very low and no other scattering except that from the wire point targets. The experiments were conducted with the parameters shown in Table 1. 
Table 1: Experimental Parameters

\begin{tabular}{|c|c|}
\hline Properties & Values \\
\hline Speed of Sound in Water, $\mathrm{m} / \mathrm{s}$ & 1482 \\
\hline Attenuation Water, dB/MHz/cm & 0.002 \\
\hline Number of Elements & 128 \\
\hline Transducer Centre Frequency, $\mathrm{MHz}$ & 4.79 \\
\hline -6 dB Transducer Bandwidth, $\%$ & 57 \\
\hline Transducer Element Pitch, $\mathrm{mm}$ & 0.3048 \\
\hline Sampling Frequency, Tx/Rx, MHz & $160 / 80$ \\
\hline Excitation Signal & 2-cycle Sinusoid \\
\hline
\end{tabular}

\subsection{Ultrasound Array Research Platform II (UARP II)}

The UARP II is a custom ultrasound imaging system developed by the Ultrasonics and Instrumentation Group at the University of Leeds $[3,4,15,16,17,18]$. It contains an 8field programmable gate array (FPGA) backplane which connects a computer running any 64-bit version of the Windows operating system (OS) via a peripheral component interconnect express (PCIe) link. Each FPGA card consists of 16 channels and there are total of 128 channels in the current UARP II. However, the design of this system is highly flexible, and the system can be easily scaled to have more channels. All excitation signals except the square pulse are designed in the Matlab software (The MathWorks Inc., Natick, MA, USA) by using a harmonic reduction pulse width modulation (HRPWM) method [15]. The sampling rate for Tx is $160 \mathrm{MHz}$. Those signals are then uploaded to the UARP II which excites the connected probe by using a five-level switched mode excitation scheme [3]. The received radio frequency $(\mathrm{RF})$ data are acquired at an 80$\mathrm{MHz}$ sampling rate and processed off line using MATLAB. The maximum sampling depth for a single firing can be more than 32768 samples per channel, which equates to a round trip in water of approximately $61 \mathrm{~cm}$ with the speed of sound of $1482 \mathrm{~m} / \mathrm{s}$ at $22^{\circ} \mathrm{C}$.

\subsection{Beamforming}

The wire targets were imaged with PWI and beamformed with the delay-and-sum (DAS) technique. The B-mode image was log-compressed and displayed with a dynamic range of $50 \mathrm{~dB}$. The B-mode image was formed point by point, $\left(x_{f}, z_{f}\right)$ with different sets of time delays assigned to the same received RF signals according to equation [19]:

$$
\tau_{i}\left(x_{f}, z_{f}\right)=\frac{z_{f} \cos \left(\theta_{n}\right)+x_{f} \sin \left(\theta_{n}\right)+\frac{L}{2} \sin \left(\theta_{n}\right)}{c}
$$

where $i=1,2,3 \ldots 128, L$ is the aperture, $\theta_{n}$ is the steering angle and $n$ is steered plane waves. In this study a single plane wave was employed, and thus, $\theta_{n}=0^{\circ}$ and $n=1$. The computed time delays were added to the received RF signal $s_{i}(t)$, giving the aligned RF signal $v_{i}(t)$, which can be represented by the following equation [20]:

$$
v_{i}(t)=s_{i}\left(t-\tau_{i}\left(x_{f}, z_{f}\right)\right)
$$

The phase deviation between the aligned RF signals, $v_{i}(t)$ on all the elements were calculated by applying crosscorrelation techniques relative to the RF signals from element $1, v_{1}(t)$ according to the following equation:

$$
p_{v v i}(\tau)=\sum_{t=t 1}^{t 2} v_{1}(t) v_{i}(t+\tau)
$$

Where $t_{1}=40 \mu \mathrm{s}, t_{2}=43.75 \mu \mathrm{s}$. The time duration equivalent to the total number of 300 or 6000 samples for the sampling frequencies of $80 \mathrm{MHz}$ and $1600 \mathrm{MHz}$ respectively. The time period selected as the wire targets located at the $20 \mathrm{~mm}$ depth.

The lag value between the $p_{v v i}(\tau)$ signal peaks to the origin of the signal, $p_{v v i}(\tau=0)$ determine the phase deviation between the RF signals.

\section{RESULTS}

The aligned RF signals for the imaging line located at $x_{f}=$ $0.5 \mathrm{~mm}$ as shown in Figure 2 for the wire target at the $20 \mathrm{~mm}$ depth have been used to analyze the effect of phase errors.

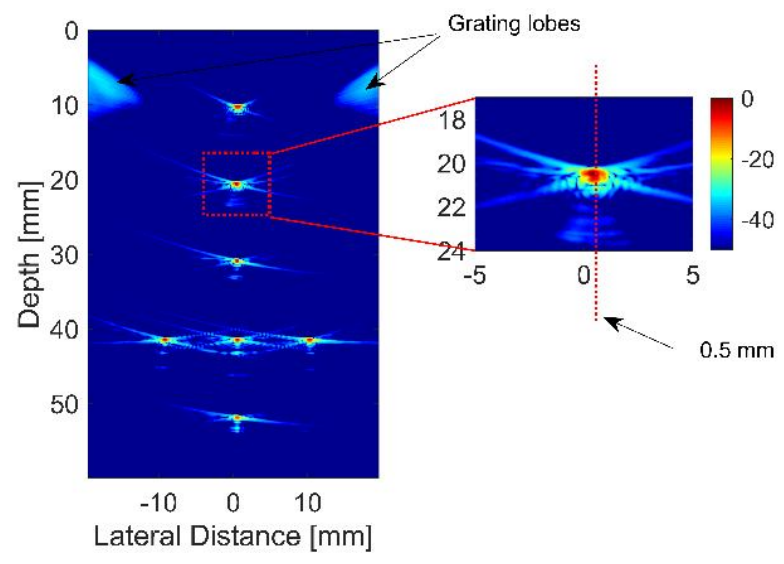

Figure 2: B-mode image of point targets beamformed with DAS obtained with PWI. The red dashed line marked on the point target at $20 \mathrm{~mm}$ depth shows the imaging line chosen for phase error analysis.

In an ideal case, aligning the RF signals for the central imaging line, for a wire target located at the center of the imaging medium, is expected to produce a set of RF signals in a straight-line form parallel to the face of the transducer. Thus, any deviation in the aligned RF signals can be considered as the result of phase error. A portion of the aligned RF signals obtained for the wire target at the $20 \mathrm{~mm}$ depth is shown in Figure 3(a). It can be seen that the signals 
were not perfectly straight (aligned) and still maintained the parabolic shape of the Rx echo signal. Figure 3(b) shows the RF signals from the elements 1, 64 and 128. Qualitatively, all of the RF signals on those elements were not in-phase with each other. The RF signals between elements 1 and 64 have a phase shift of approximately $76^{\circ}$ and $38^{\circ}$ between elements 1 and 128.
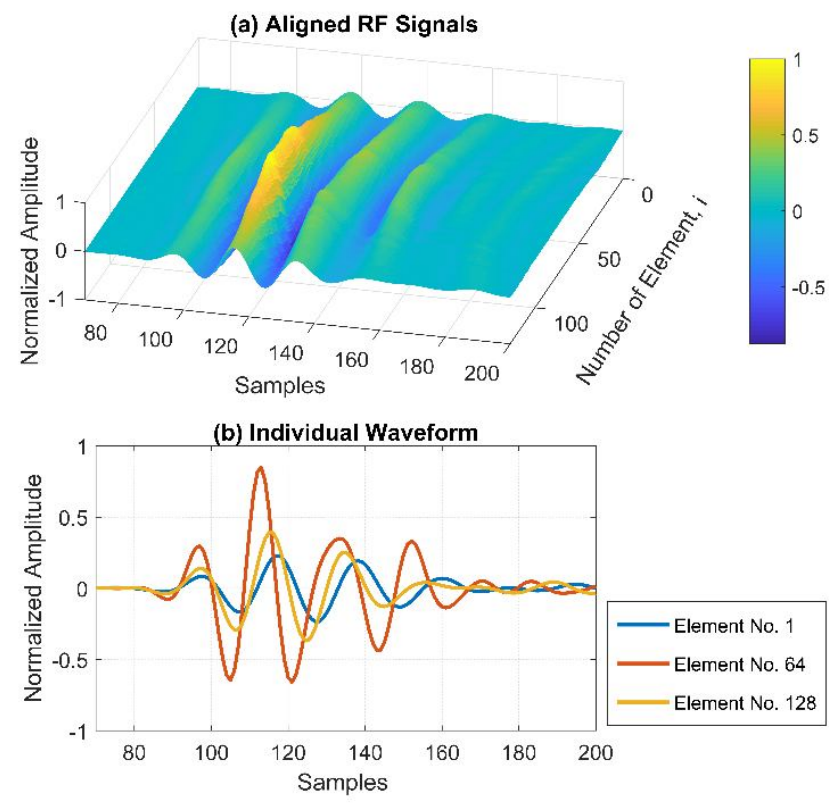

Figure 3: a) Aligned RF signals for the imaging line at $x_{f}=$ $0.5 \mathrm{~mm}$ at the $20 \mathrm{~mm}$ depth and b) individual waveforms from elements 1,64 and 128 .

The phase error was computed with two different sampling frequencies. The first is the UARP II hardware Rx sampling frequency of $80 \mathrm{MHz}$ and the second is a higher sampling frequency of $1600 \mathrm{MHz}$ produced by up-sampling the time domain signal $v_{i}(t)$, using the linear interpolation technique. The results obtained with the higher sampling frequency provided more details on the phase error compared to that with the lower sampling frequency. The accurate estimated phase error helps to determine more precisely the number of samples needed to align the RF signals. The result is shown in Figure 4.

The maximum and total phase errors for a single imaging line calculated for 128 elements with the sampling frequencies of $80 \mathrm{MHz}$ and $1600 \mathrm{MHz}$ are given in Table 2 . The higher sampling frequency provides more accurate results than the lower sampling frequency. The time calculated with a higher frequency shows an increase of 6.26 $\%$ from $50 \mathrm{~ns}$ to $53.13 \mathrm{~ns}$, while the distance shows an increase of $6.4 \%$ from $37 \mu \mathrm{m}$ to $39.37 \mu \mathrm{m}$. This is because the interpolation function can increase the accuracy by adding the missing data [21-25].
Once the phase error represented by the lag values was calculated, a sign-reversed lag value was applied to the aligned RF signals in order to compensate for the aberration. Figure 5 shows the results before and after phase correction takes place on the wire target at $20 \mathrm{~mm}$ depth.

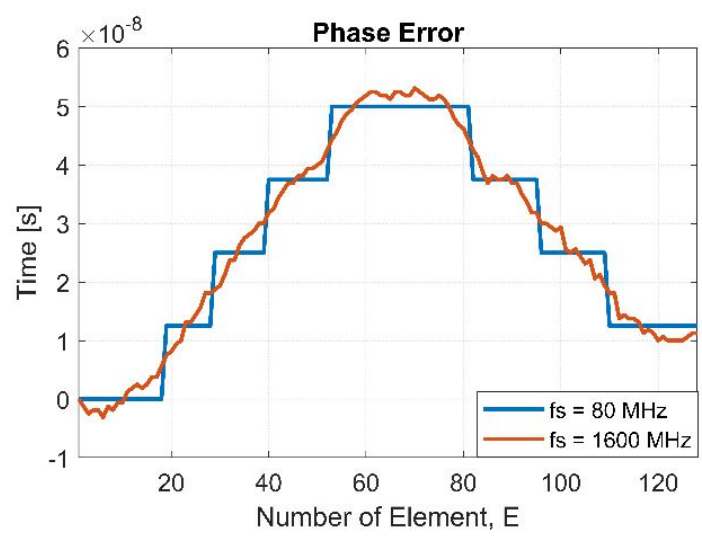

Figure 4: Phase errors measured on the aligned RF signals relative to the first element for the imaging line at $x_{f}=0.5$ $\mathrm{mm}$ in the lateral direction as shown in Figure 2.

Table 2: Phase Error

\begin{tabular}{|c|c|c|c|c|}
\hline & \multicolumn{2}{|c|}{$80 \mathrm{MHz}$} & \multicolumn{2}{c|}{$1600 \mathrm{MHz}$} \\
\cline { 2 - 5 } & $\begin{array}{l}\text { Time, } \\
n s\end{array}$ & $\begin{array}{l}\text { Distance, } \\
\mu m\end{array}$ & $\begin{array}{l}\text { Time, } \\
n s\end{array}$ & $\begin{array}{l}\text { Distance, } \\
\mu m\end{array}$ \\
\hline $\begin{array}{c}\text { Maximum } \\
\text { Phase } \\
\text { Error }\end{array}$ & 50 & 37 & 53.13 & 39.37 \\
\hline $\begin{array}{c}\text { Total } \\
\text { Phase } \\
\text { Error }\end{array}$ & 3450 & 3560 & 3462.5 & 2565.7 \\
\hline
\end{tabular}

Two different regions, $\mathrm{A}$ and $\mathrm{B}$ have been chosen on the $\mathrm{B}$ mode image to highlight the noise pattern changes according to the sampling rate. In region A, more side lobes and clutter noise are produced on both sides of the wire target when the lower sampling rate, $80 \mathrm{MHz}$ was used to correct the phase error. This means the compensated RF signals are still not properly aligned. Correcting the phase aberration with both low and high sampling frequencies can reduce noise in the lateral direction. This has been highlighted in region B in Figure 5.

As shown in Figure 6, the main lobe in the lateral direction has been improved when the aligned RF signals are compensated with both lower and higher frequencies. The lateral resolution at $-6 \mathrm{~dB}$ and $-20 \mathrm{~dB}$ showed improvements from $0.42 \mathrm{~mm}$ to $0.34 \mathrm{~mm}$ and from $1.18 \mathrm{~mm}$ to $0.56 \mathrm{~mm}$, respectively. No significant changes occurred on the axial main lobe. However, the peak side lobes (PSLs) have increased from $-30 \mathrm{~dB}$ to $-20 \mathrm{~dB}$ after the correction. The small amount of the increment (from $-26 \mathrm{~dB}$ to $-24 \mathrm{~dB}$ ) in PSL can be also seen in the axial direction. 

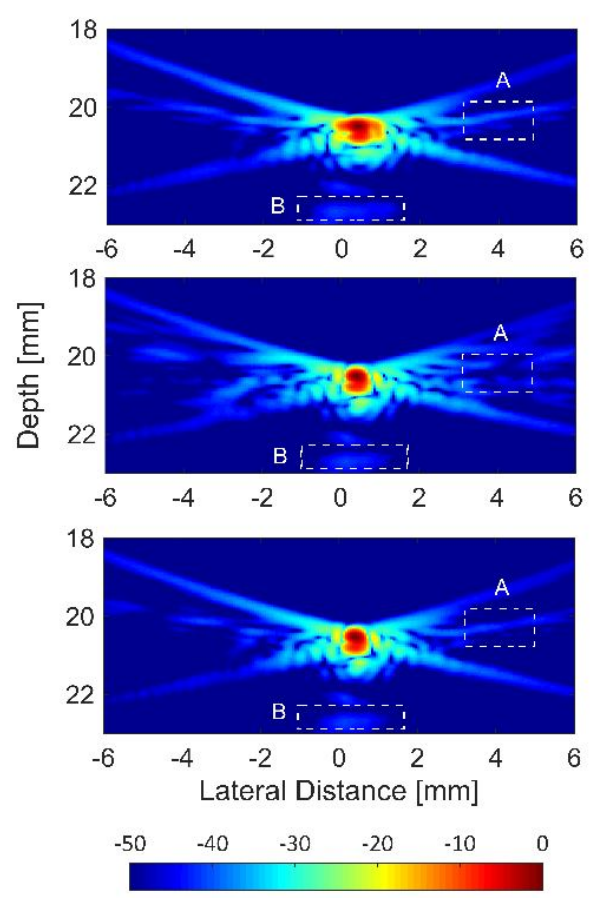

Figure 5: B-mode image of the wire target at $20 \mathrm{~mm}$ depth with phase error (Top), after phase error correction with the sampling rate of $80 \mathrm{MHz}$ (Middle) and after phase error correction with the sampling rate of $1600 \mathrm{MHz}$ (Bottom). The dashed boxes, marked with $\mathrm{A}$ and $\mathrm{B}$, show the effect of phase correction on noisy regions. (a) Axial Beam Profile
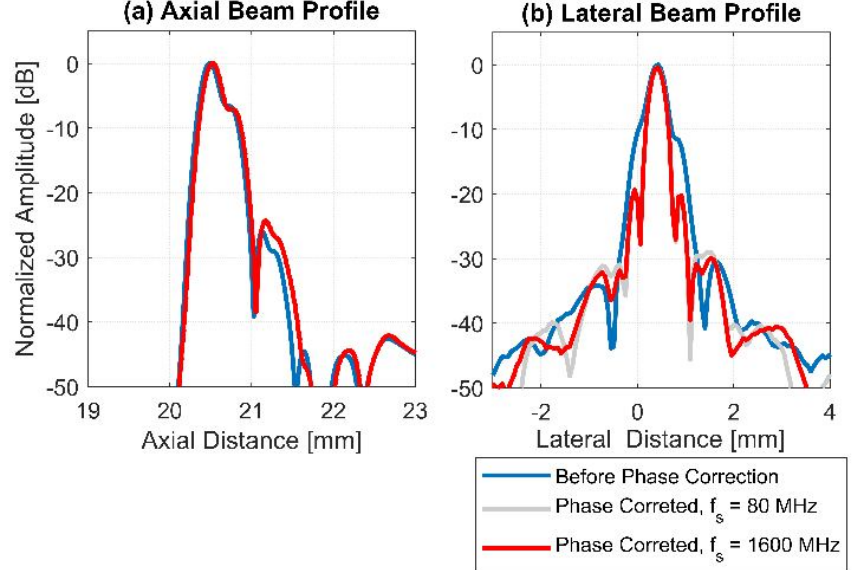

Figure 6: Beam profiles of the wire target at $20 \mathrm{~mm}$ depth in the a) axial direction and b) lateral direction before and after correction of phase errors.

\section{CONCLUSION}

The phase error that occurs due to the non-ideal transducer characteristic can be overcome by adding a sign-reversed lag to the aligned RF signals. Higher sampling rate signals are able to produce the same lateral resolution as low sampling signals. However, noise that is present on the B-mode image is much less with a higher sampling rate. The phase corrected signal can improve the signal resolution in the lateral direction and reduce the noise level. On the other hand, the side lobes are not eliminated and are still visible within the imaging region.

\section{ACKNOWLEDGEMENT}

Part of this work was financially supported by Ministry of Higher education, Malaysia (Grant No. FRGS/1/2019/TK04/UKM/03/4). Funders were not involved in the conduct of the research.

\section{REFERENCE}

1. JM Hansen. Synthetic Aperture Compound Imaging. Ph.D. dissertation, Technical University of Denmark (DTU), Denmark, 2012.

2. Hopp, T., M. Zapf, H. Gemmeke, and N. V. Ruiter. Experimental evaluation of straight ray and bent ray phase aberration correction for USCT SAFT imaging. International Society for Optics and Photonics in Medical Imaging 2018: Ultrasonic Imaging and Tomography, vol. 10580, p. 105800M., 2018. https://doi.org/10.1117/12.2292895

3. Cowell DMJ, Freear S. Separation of Overlapping Linear Frequency Modulated (LFM) Signals Using the Fractional Fourier Transform. IEEE Transactions on Ultrasonics, Ferroelectrics, and Frequency Control, vol 57, no 10, pp. 2324-2333. 2010.

4. Cowell DMJ, Freear S. Quinary excitation method for pulse compression ultrasound measurements. Ultrasonics, vol 48, no 2, pp. 98108. 2008.

5. Moubark, A. M., Sevan Harput, Cowell DMJ, Steven Freear. Clutter noise reduction in B-Mode image through mapping and clustering signal energy for better cyst classification. IEEE International Ultrasonics Symposium (IUS), pp. 1-4. IEEE, 2016. https://doi.org/10.1109/ULTSYM.2016.7728860

6. Moubark, A. M., Alomari, Z,Sevan Harput, Steven Freear. Comparison of spatial and temporal averaging on ultrafast imaging in presence of quantization errors. IEEE International Ultrasonics Symposium (IUS), pp. 1- 4. IEEE, 2015.

7. Alshaya, A., Sevan Harput, Moubark, AM, Cowell DMJ, McLaughlan J., Steven Freear. Spatial resolution and contrast enhancement in photoacoustic imaging with filter delay multiply and sum beamforming technique. IEEE International Ultrasonics Symposium (IUS), pp. 14. IEEE, 2016.

8. Moubark, A. M., Cowell DMJ, Sevan Harput, Steven Freear. New Denoising Unsharp Masking Method for Improved Intima Media Thickness Measurements with Active Contour Segmentation. IEEE International Ultrasonics Symposium (IUS), pp. 1- 4. IEEE, 2018.

9. Md Ashikuzzaman, Clyde Belasso, Claudine J. Gauthier, Hassan Rivaz. Suppressing Clutter Components In Ultrasound Color Flow Imaging Using Robust Matrix Completion Algorithm: Simulation And Phantom 
Study. IEEE International Symposium on Biomedical Imaging (ISBI), pp. 1-4. IEEE, 2019.

10. Bayat, Mahdi, and Mostafa Fatemi. Concurrent clutter and noise suppression via low rank plus sparse optimization for non-contrast ultrasound flow doppler processing in microvasculature. IEEE International Conference on Acoustics, Speech and Signal Processing (ICASSP), pp. 1080-1084. IEEE, 2018.

11. Huang, Chengwu, Pengfei Song, Ping Gong, Joshua D. Trzasko, Armando Manduca, and Shigao Chen. Debiasing-based Noise Suppression for Ultrafast Ultrasound Microvessel Imaging. IEEE transactions on ultrasonics, ferroelectrics, and frequency control, vol 68, no 8, pp. 1281-1291, 2019.

12. Solomon, Oren, Regev Cohen, Yi Zhang, Yi Yang, Qiong He, Jianwen Luo, Ruud JG van Sloun, and Yonina C. Eldar. Deep unfolded robust pca with application to clutter suppression in ultrasound. IEEE transactions on medical imaging, 2019.

13. Bhanuprakash Dudi, Rajesh V. Medicinal Plant Recognition based on CNN and Machine Learning. International Journal of Advanced Trends in Computer Science and Engineering (IJATCSE), ISSN 2278 - 3091, vol. 8, no. 4, July-August 2019. https://doi.org/10.30534/ijatcse/2019/03842019

14. Nock, Levin and Trahey, Gregg E and Smith, Stephen W. Phase aberration correction in medical ultrasound using speckle brightness as a quality factor. The Journal of the Acoustical Society of America, vol 85, no 5, 1819-1833, 1989.

15. Smith PR, Cowell DMJ, Freear S. Width-modulated square-wave pulses for ultrasound applications. IEEE Transactions on Ultrasonics, Ferroelectrics, and Frequency Control, vol 60, no 11, pp. 2244-2256, 2013.

16. Cowell DMJ, Smith PR; Freear S. Phase-inversionbased selective harmonic elimination (PI-SHE) in multi-level switched-mode tone-and frequencymodulated excitation. IEEE Transactions on Ultrasonics, Ferroelectrics, and Frequency Control, vol 60, no 6, pp. 1084-1097, 2013.

17. Smith PR, Cowell DMJ; Raiton B; Vo Ky C; Freear S. Ultrasound array transmitter architecture with high timing resolution using embedded phase-locked loops. IEEE Transactions on Ultrasonics, Ferroelectrics and Frequency Control, vol 59, no 1, pp. 40-49, 2013.

18. S. Freear, D. Cowell, and P. Smith, "Ultrasound generation," Jun. 26, 2014, WO Patent App. PCT/GB2013/053,289.

19. Moubark, AM., Alomari, Z., Harput, S., Cowell, D. M., \& Freear, S. Enhancement of contrast and resolution of B-mode plane wave imaging (PWI) with non-linear filtered delay multiply and sum (FDMAS) beamforming. IEEE International Ultrasonics Symposium (IUS), pp. 1-4, 2016.

20. Moubark, Asraf Mohamed, Thomas M. Carpenter, Cowell DMJ, Sevan Harput, and Steven Freear. New improved unsharp masking methods compatible with ultrasound B-mode imaging. IEEE International Ultrasonics Symposium (IUS), pp. 1-4. IEEE, 2017.
21. Mahmoudzadeh, Amir Pasha, and Nasser H. Kashou. Evaluation of interpolation effects on upsampling and accuracy of cost functions-based optimized automatic image registration. Journal of Biomedical Imaging. pp 16, 2013.

22. Kim, Jiwon, Jung Kwon Lee, and Kyoung Mu Lee. Accurate image super-resolution using very deep convolutional networks. IEEE conference on computer vision and pattern recognition, pp. 1646-1654. 2016. https://doi.org/10.1109/CVPR.2016.182

23. Narayan Gupta, Devanand. Removal of Various noise Signals from Medical Images Using Wavelet Based Filter and Unsymmetrical Trimmed Median Filter. International Journal of Computer Science and Mobile Computing, vol 4, no 4, pp. 684-690, 2015.

24. Narayan Gupta, Devanand. Removal of Various noise Signals from Medical Images Using Wavelet Based Filter and Unsymmetrical Trimmed Median Filter. International Journal of Computer Science and Mobile Computing, vol 4, no 4, pp. 684-690, 2015.

25. Navneet Malik, Nilesh N Wani, Jimmy Singla. Complications of Sight Threatening Diabetic Retinopathy: A Review. International Journal of Advanced Trends in Computer Science and Engineering (IJATCSE), ISSN 2278 - 3091, vol. 8, no. 4, July-August 2019.

https://doi.org/10.30534/ijatcse/2019/09842019 